\title{
Correction: Adaptive optics: principles and applications in ophthalmology
}

\author{
Engin Akyol $\mathbb{D} \cdot$ Ahmed M. Hagag $(\mathbb{D} \cdot$ Sobha Sivaprasad • Andrew J. Lotery $\mathbb{D}$
}

Published online: 7 January 2021

(C) The Author(s), under exclusive licence to The Royal College of Ophthalmologists 2020

Correction to: Eye

https://doi.org/10.1038/s41433-020-01286-z

Since the online publication of the paper above, the authors have noticed that the titles and captions of Figs. 5 and 6 were interchanged. The corrected titles/captions should read:

Fig. 5 Adaptive optics scanning laser ophthalmoscope (AO-SLO) imaging of photoreceptors in a healthy subject and diabetic patients. a, c, e Cone reflection images. $\mathbf{b}, \mathbf{d}, \mathbf{f}$ Voronoi analysis of cone photoreceptors in $(\mathbf{a}, \mathbf{c}, \mathbf{e})$, respectively. Upper row: densely packed cone photoreceptors in a healthy control subject. Middle and lower rows: reduced cone density and increased irregularity in the cone mosaic in patients with diabetic retinopathy. Reprinted with permission from Lammer et al. [152].
Fig. 6 Photoreceptor imaging in the left eye of a 71-year old female patient with intermediate age-related macular degeneration. a A 30-degree en face infrared image with overlaid 4-degree adaptive optics flood illumination ophthalmoscope (AO-FIO) image (inside the yellow square). $\mathbf{b}$ The enlarged AO-FIO image of cone photoreceptor mosaic (4 degrees temporal to the centre of the fovea). The area surrounded by a yellow square was enlarged for the quantitative analysis of cones. c Semiautomated detection of photoreceptors. Red squares correspond to automatically identified cones. Green squares represent cones that were manually corrected/identified. d-f Voronoic colour-coded maps of cone density, spacing, and regularity, respectively.

The authors sincerely apologise for the error and any inconvenience it might have caused. 